\title{
Revisiting Turbulence Model Validation for High-Mach Number Axisymmetric Compression Corner Flows
}

\author{
Nicholas J. Georgiadis*, \\ NASA Glenn Research Center, Cleveland, OH 44135 \\ Christopher L. Rumsey ${ }^{\dagger}$, \\ NASA Langley Research Center, Hampton, VA 23681 \\ George P. Huang \\ Wright State University, Dayton, OH, 45345
}

\begin{abstract}
Two axisymmetric shock-wave / boundary-layer interaction (SWBLI) cases are used to benchmark one- and two-equation Reynolds-averaged Navier-Stokes (RANS) turbulence models. This validation exercise was executed in the philosophy of the NASA Turbulence Modeling Resource and the AIAA Turbulence Model Benchmarking Working Group. Both SWBLI cases are from the experiments of Kussoy and Horstman for axisymmetric compression corner geometries with SWBLI inducing flares of 20 and 30 degrees, respectively. The freestream Mach number was approximately 7 . The RANS closures examined are the Spalart-Allmaras one-equation model and the Menter family of $k-\omega$ two equation models including the Baseline and Shear Stress Transport formulations. The Wind-US and CFL3D RANS solvers are employed to simulate the SWBLI cases. Comparisons of RANS solutions to experimental data are made for a boundary layer survey plane just upstream of the SWBLI region. In the SWBLI region, comparisons of surface pressure and heat transfer are made. The effects of inflow modeling strategy, grid resolution, grid orthogonality, turbulent Prandtl number, and code-to-code variations are also addressed.
\end{abstract}

\section{Nomenclature}

$a_{1} \quad$ coefficient in SST model used to limit turbulent shear stress

$C_{p} \quad$ specific heat at constant pressure

$F_{2} \quad$ SST model switching function

$k$ turbulent kinetic energy

$M_{\infty} \quad$ local freestream Mach number

$\mathcal{P}_{k} \quad$ turbulent production

$P_{s} \quad$ static pressure

$\mathrm{Pr}_{t} \quad$ turbulent Prandtl number

$Q_{w} \quad$ wall heat flux

$S \quad$ coordinate measured along surface of cylinder and flare

$S_{i j} \quad$ strain rate tensor

$T_{s} \quad$ static temperature

$T_{t} \quad$ stagnation temperature

$T_{w} \quad$ wall temperature

$U_{\infty} \quad$ local freestream velocity

$u_{i} \quad$ velocity vector

*Aerospace Engineer, Inlets and Nozzles Branch, Associate Fellow AIAA.

${ }^{\dagger}$ Aerospace Engineer, Computational AeroSciences Branch, Fellow AIAA.

${ }^{\ddagger}$ Professor and Chair, Associate Fellow AIAA. 


$\begin{array}{ll}W_{i j} & \text { vorticity tensor } \\ x, y, z & \text { Cartesian coordinates } \\ X_{t} & \text { transition specification location } \\ y^{+} & \text {normal distance in wall coordinates } \\ \delta & \text { boundary layer thickness } \\ \delta_{v} & \text { velocity thickness } \\ \delta^{*} & \text { displacement thickness } \\ \mu_{t} & \text { dynamic eddy viscosity } \\ \nu & \text { kinematic molecular viscosity } \\ \tilde{\nu} & \text { turbulence field variable in Spalart-Allmaras model } \\ \omega & \text { turbulent dissipation rate } \\ \Omega & \text { vorticity magnitude } \\ \rho & \text { density } \\ \tau_{i j}^{T} & \text { turbulent stress tensor } \\ \tau_{w} & \text { wall shear stress } \\ \theta & \text { momentum thickness, also flare angle in Fig. } 1 \text { taken from Ref. } 5 .\end{array}$

\section{Introduction}

Despite the importance to the aerodynamic community of identifying, understanding, and controlling separated flows, the numerical prediction of flow separation remains elusive. In supersonic flows, the shock-wave / boundary-layer interaction (SWBLI) produces especially complex flow separations that have significant effects on the operability of specific components such as aircraft inlets and also on overall performance of aerospace vehicles. A recent workshop considering Computational Fluid Dynamics (CFD) calculations for a set of SWBLI cases was held in conjunction with the 48th American Institute of Aeronautics and Astronautics (AIAA) Aerospace Sciences Meeting. The focus of this workshop ${ }^{1,2}$ was SWBLI as occurs in supersonic inlets. Several investigators contributed solutions to this workshop including both Reynolds-averaged NavierStokes (RANS) computations and Large-Eddy Simulations (LES) computations. DeBonis et al. ${ }^{3}$ provided a comprehensive assessment of the CFD calculations, including uncertainty analysis of the submitted CFD results and experimental data obtained for the same configurations. The results of the workshop indicated that none of the RANS methods were able to capture the details of the SWBLIs and that LES, while offering promise for the future, still cannot be applied to configurations at realistic Reynolds numbers.

Most of the new research in computational turbulence is associated with LES, hybrid RANS-LES, and Direct Numerical Simulations (DNS). There is some renewed interest in making RANS improvements, where possible, although the expected gains are not substantial. An effort sponsored by the AIAA Fluid Dynamics Technical Committee (FDTC) in recent years has focused on benchmarking RANS turbulence models. ${ }^{4}$ The AIAA Turbulence Model Benchmarking Working Group (TMBWG) has established a website, referred to as the NASA Turbulence Modeling Resource, with several test cases and model descriptions. The test cases use well established data and carefully constructed CFD models. The grids utilized for each case must achieve near grid-independence. Boundary conditions are provided in unambiguous detail. Also, a "model readiness" rating system indicates the maturity of turbulence models in terms of the number of codes they have been implemented in. There are several benefits of this effort of which two primary ones are: (1) a common benchmarking of the models is presented, where code developers may determine if their implementation of a given model is the same as published and programmed into other codes, and (2) accuracy and shortcomings of existing models may be demonstrated, thereby providing motivation for newer development efforts. These development efforts may be new or enhanced RANS models as is the focus of the NASA Turbulence Modeling Resource, but may also extend to the direct calculation methods such as DNS, LES, and hybrid RANS-LES.

The work described in this report consists of RANS benchmarking of a class of flows not previously considered by the TMBWG, that of a high Mach number SWBLI. The NASA Turbulence Modeling Resource considers a large number of subsonic flows, some with flow separations, but the only cases at supersonic Mach numbers are a flat plate boundary layer and square duct flow. The specific cases considered here are from the axisymmetric compression corner experiments of Kussoy and Horstman. ${ }^{5}$ These cases were previously considered by Huang and Coakley ${ }^{6}$ and Olsen et al. ${ }^{7}$ Their efforts examined the performance of standard turbulence models and additional models with corrections to improve separation prediction. This 
paper presents results for two cases that come from the Kussoy-Horstman experiments. The first case is a 20-degree compression corner and the second case is a 30-degree compression corner. The objective is to benchmark commonly used RANS models for high Mach number SWBLI flows. Grid sensitivity, inflow boundary treatment, and turbulence model sensitivities are presented. Two codes were used in this effort: Wind-US ${ }^{8,9}$ and CFL3D. ${ }^{10}$

\section{Flow Cases}

In Ref. 5, Kussoy and Horstman investigated a series of axisymmetric compression corner experiments where a cone/ogive cylinder forebody had flares of varying angles (20 degrees to 35 degrees). As found in the previously cited AIAA SWBLI workshop, many nominally two-dimensional experiments are complicated by sidewall effects. In fact, the sidewall interactions have frequently been found to be more substantial than the interaction that is the focus of the experiments. These sidewall effects also frequently influence the SWBLI in the focus region. Axially symmetric experiments such as those of Ref. 5 eliminate the three-dimensional complications and also enable comparatively easier instrumentation, in terms of placement of probes and surface measurements. Computationally, RANS may be run in axisymmetric mode, which simplifies grid generation and also lowers the computational cost relative to truly three-dimensional configurations. Of course methods such as LES and DNS would still require three-dimensional computations for turbulent flows.

A schematic of the test article used in the experiments of Kussoy and Horstman is shown in Fig. 1. The leading edge is a 10-degree cone that transitions via a circular arc to a constant area cylinder. A flare designed to produce an oblique shock and SWBLI is placed at a location $139 \mathrm{~cm}$ downstream of the leading edge. As the angle of the flare increases, the strength of the oblique shock and the size of the SWBLI region both increase. The boundary layer was surveyed via pitot pressure and stagnation temperature probes just upstream of the interaction region, at a position $6 \mathrm{~cm}$ upstream of the flare. The quantities measured in the interaction region were wall surface pressure and surface heat transfer, the latter which was obtained by the transient thin-skin technique described in Ref. 5. Because of the initial shock from the cone leading edge, the local freestream conditions outside the boundary layer and just before the flare were found to be the following: $M_{\infty}=7.05, T_{s}=81.2 \mathrm{~K}, P_{s}=576 \mathrm{~Pa}, U_{\infty}=1274 \mathrm{~m} / \mathrm{s}$. These conditions correspond to a Reynolds number of $57000 / \mathrm{cm}$. The 20-degree flare case is examined first in this paper and then the 30degree flare. Most of the computational variations were conducted for the 20-degree case. Two approaches to modeling the geometry of the case resulted in different upstream inflow conditions needed to match the local freestream conditions and are discussed in the next section.

\section{Computational Details}

\section{Modeling Approaches}

Two geometric modeling approaches were used in this effort. The first considered the entire geometry from the leading edge of the cone, over the cylinder and then over the flare. In the experiments, transition was believed to occur at a position just downstream of where the constant area cylinder started, so multiple transition specification locations were examined as part of this work with the goal of trying to match the boundary layer measurements taken at $S=-6 \mathrm{~cm}$. The second modeling approach did not include the entire cone and forebody and no point transition was specified. Instead, the inflow was placed at a position along the constant area cylinder with the run of axial length selected to again try to match the boundary layer measured in the experiments at $S=-6 \mathrm{~cm}$. The first approach is termed "full geometry" in the rest of this paper while the shortened geometry is referred to as "no cone."

The axisymmetric computational grids used for these two approaches (20-degree case) are illustrated in Fig. 2. The full geometry grid consisted of 961 axial points by 401 vertical points. The no-cone grid had 641 axial points by 401 vertical points. CFL3D represented the axisymmetric grid by rotating the grid 1 degree in the azimuthal direction to create a second plane. Periodic boundary conditions were employed in the azimuthal direction on these two planes. Wind-US uses a single plane and a two-dimensional grid with an angle set by default to 5 degree to compute metric terms enabling an axisymmetric computation. Figure 3 shows more details of the computational grid utilized near the SWBLI focus region. The region from the $S=-6 \mathrm{~cm}$ station to the outflow had 321 axial points. Note that the grids for the two approaches were 
identical in this region; they only differed upstream. For an axisymmetric RANS analysis, these grids are of rather high density compared to what may be considered standard for an everyday engineering analysis. They were designed to be very dense in accordance with other validation cases on the NASA Turbulence Modeling Resource website, and with grid sensitivity issues fully addressed. The position of the first point off of the wall was placed such that the $y^{+}$at the upstream boundary layer measurement station was 0.05 on the finest grid level. After the SWBLI, the same physical spacing was found to correspond to a $y^{+}$of 0.5 which is due to a steep increase in wall shear stress after the interaction. The grids were also manually adapted to the shocks in the flow. For the full-geometry case, there is an oblique shock from the cone leading edge and another back at the flare, while for the no-cone case there is a weak interaction at the inflow, and an oblique shock at the cylinder-flare intersection.

In order to achieve the local freestream conditions discussed previously, the inflow boundary conditions for the two modeling approaches differed. Freestream foundry conditions were set at the inflow and top boundary. Extrapolation was used at the outflow. For the full-geometry case, the inflow Mach number was set as 7.062, the inflow stagnation pressure was $2839 \mathrm{kPa}$ and the inflow stagnation temperature was 888.9 K. For the case with no cone, the inflow Mach number was set as 7.11, the inflow stagnation pressure was $2515 \mathrm{kPa}$ and the inflow stagnation temperature was $888.9 \mathrm{~K}$. At first impression, it may have been anticipated that the inflow Mach number needed for the full-geometry approach would be higher than for the no-cone approach, but after the 10-degree cone and associated oblique shock, the flow does turn back to the axial direction and expands to nearly an identical local freestream Mach number (although reduced stagnation pressure). For the no-cone approach, there is a small, but not negligible wave originating from the leading edge of the domain, which reduces the Mach number from 7.11. All of the CFD results discussed in this paper enable agreement with the experimentally measured local freestream conditions (outside of the boundary layer) for Mach number, axial velocity, static pressure, and static temperature to under 0.5 percent.

As discussed in an earlier paragraph, transition was anticipated to occur near the start of the constant area cylinder. In the full-geometry approach, a few different transition specification locations were used (with the Menter Shear Stress Transport model) in order to try to match the integral thicknesses at $S=-6$ $\mathrm{cm}$. For all cases, the wall temperature was set to $311 \mathrm{~K}$, as was done in the experiments.

\section{Turbulence Modeling}

Of the RANS turbulence models applied to the SWBLI cases in the previously described AIAA workshop, the most commonly used model by the participants was the two-equation Shear Stress Transport (SST) model of Menter. ${ }^{11}$ The SST model is a $k-\omega$ formulation that has become one of the most widely used turbulence models for RANS analyses. For small separations due to mild adverse pressure gradients in subsonic flows, the model has demonstrated success. As observed in the results of the workshop and elsewhere, however, the SST model tends to overpredict the size of flow separations in SWBLI problems. A second turbulence model, the one-equation turbulence model of Spalart and Allmaras ${ }^{12,13}$ (SA), was also used by a number of investigators in the aforementioned workshop. The SST and SA models are two of the most widely used turbulence models in the aerodynamics and broader flow analysis community. These two models have also been used for every case that is already included in the NASA Turbulence Modeling Resource website.

In Ref. 11, Menter also introduced a similar $k-\omega$ model without a stress limiter, called the baseline (BSL) model, which in contrast to its sister model SST, tends to underpredict the magnitude of shockinduced separations. A third variant of the Menter $k-\omega$ turbulence model family was employed here where the coefficient associated with the shear stress limiter, termed $a_{1}$, was raised from the default value of 0.31 to 0.355. This coefficient limits the modeled shear stress to not exceed the turbulent kinetic energy in the inner three-fourths of the boundary layer by a prescribed fraction. This is accomplished via the eddy viscosity expression, shown in Eqn. 1.

$$
\mu_{t}=\frac{\rho a_{1} k}{\max \left(a_{1} \omega, F_{2} \Omega\right)}=\min \left(\frac{\rho k}{\omega}, \frac{\rho a_{1} k}{F_{2} \Omega}\right)
$$

The default setting for $a_{1}$ was 0.31 in Menter ${ }^{11}$ based primarily on subsonic experience. However, in Ref. 14, examination of experimental data and computational solutions for SWBLI problems indicated that a larger value for $a_{1}$ may yield improved predictions for some SWBLI dominated flows. 
An additional factor for all of the $k-\omega$ models considered here is the production source term used in both the $k$ and $\omega$ equations. The standard version of the model as reported on the NASA Turbulence Modeling Resource website and in Ref. 11 uses the rate of strain in the production term. This is shown in Eqns. 2 and 3. On the website and in this report, this form of the models will be denoted as "SST" and "BSL."

$$
\mathcal{P}_{k}=\tau_{i j}^{T} \frac{\partial u_{i}}{\partial x_{j}}
$$

where

$$
\tau_{i j}^{T}=\mu_{t}\left(2 S_{i j}-\frac{2}{3} \frac{\partial u_{k}}{\partial x_{k}} \delta_{i j}\right)-\frac{2}{3} \rho k \delta_{i j}
$$

and

$$
S_{i j}=\frac{1}{2}\left(\frac{\partial u_{i}}{\partial x_{j}}+\frac{\partial u_{j}}{\partial x_{i}}\right)
$$

An alternative form of the model employs the vorticity magnitude to calculate the production terms (Eqns. 5 and 6). A primary motivation for using this form is in the calculation of stagnation point flows where the shear strain rate is zero, yet strong decelerations or accelerations can lead to highly unphysical turbulent production rates. ${ }^{15}$ Usage of the vorticity form can avoid these numerically induced problems. In a zero-pressure gradient boundary layer, the two forms should yield the same turbulent production rates. The vorticity based production term models are referred to as "SST-V" and "BSL-V."

$$
\mathcal{P}_{k}=\mu_{t} \Omega^{2}-\frac{2}{3} \rho k \delta_{i j} \frac{\partial u_{i}}{\partial x_{j}}
$$

where

$$
\Omega=\sqrt{2 W_{i j} W_{i j}}
$$

and

$$
W_{i j}=\frac{1}{2}\left(\frac{\partial u_{i}}{\partial x_{j}}-\frac{\partial u_{j}}{\partial x_{i}}\right)
$$

The freestream turbulence level used with the SA model corresponded to $\tilde{\nu} / \nu=5$. For all of the Menter derived $k-\omega$ model simulations, the freestream turbulence intensity was set to $0.021 \%$ and freestream eddy viscosity ratio was set to 0.001 . The default turbulent Prandtl number, $P r_{t}$, was set to 0.9. However, a subsequent section considers sensitivity of the solutions to $\operatorname{Pr}_{t}$.

\section{0-Degree Flare Results}

This section presents several investigations where variations in the modeling approach were considered. In actually conducting these evaluations, the effects of grid resolution and inflow modeling approach were concurrently studied, but we present the inflow modeling approach first. Results comparing otherwise identical calculations (grid, turbulence model, boundary conditions) but with different codes, and effect of turbulence model selection are presented in the latter half of this section for the 20-degree flare case. 


\section{Inflow Modeling Approach}

For the full-geometry approach, only the Menter SST-V model was used with Wind-US, but different transition specification locations were examined. This was accomplished by setting a zonal boundary at the desired "transition" location and solving only laminar equations upstream of this point and RANS downstream of this point. Two-equation turbulence models like SST-V actually do mimic a transition process of sorts, such that in this case, it took $10 \mathrm{~cm}$ beyond the transition specification location for the eddy viscosity ratio in the boundary layer to exceed unity. We discuss two of these point transition sub-cases here. The first location corresponded to a position $69 \mathrm{~cm}$ downstream of the cone leading edge while the second had the transition location set at $83 \mathrm{~cm}$ downstream of the leading edge. We refer to the first as the "long $X_{t}$ " case, as it has a longer fully turbulent region, and the second as the "short $X_{t}$ " case. Figure 4 shows Mach number contours for the full-geometry case with long $X_{t}$ using the SST-V model and the shortened no-cone case that also used the SST-V model. The dominant difference between the two flows, that of the oblique shock from the cone leading edge and subsequent expansion after the turn to the constant area cylinder, is obvious from these Mach number contours. However, within the vicinity of the SWBLI, the Mach number contours are virtually identical. As a result, while Fig. 5 shows contours for the no-cone approach, the full-geometry contours are indistinguishable from these contours.

Table 1 compares integral thicknesses from the experiments to those of these computations. It may be noted that the momentum thicknesses from the computations do not agree with the experimental results. It is anticipated that obtaining the density in the experiments was a challenge as it was not directly measured but rather a derived quantity, and prone to relatively high error. As a result, we relied more heavily on the velocity thickness, because it was felt that direct comparison of this quantity would yield better agreement at the inflow station. Examining the full-geometry results, it may be observed that the "short $X_{t}$ " case matched $\delta_{v}$ more closely while the "long $X_{t}$ " case matched $\delta^{*}$ more closely.

Table 1. Comparison of inflow station $(S=-6 \mathrm{~cm}$ boundary layer state), modeling approach effects.

\begin{tabular}{lccc}
\hline Case & $\delta_{v}(\mathrm{~cm})$ & $\delta^{*}(\mathrm{~cm})$ & $\theta(\mathrm{cm})$ \\
\hline \hline Experimental Data & 0.207 & 0.735 & 0.0642 \\
SA, No Cone & 0.205 & 0.793 & 0.0526 \\
SST-V, No Cone & 0.219 & 0.762 & 0.0502 \\
SST-V, Full Geometry, Long $X_{t}$ & 0.218 & 0.740 & 0.0497 \\
SST-V, Full Geometry, Short $X_{t}$ & 0.202 & 0.638 & 0.0428 \\
\hline
\end{tabular}

The velocity profiles and stagnation temperature profiles corresponding to these cases are presented in Fig. 6. An SA model result for the no-cone case is provided for reference here, but more detailed examination of turbulence modeling effects is in a subsequent section. Compared to the SST-V results, the SA solution agrees with the experimental data best in the bottom half of the boundary layer. All of the results discussed in this section and in the previously discussed Mach number contours were for the finest computational grids. Effects of grid resolution are presented in the next section.

Figure 6 shows the effect of the differing specified transition locations on the inflow boundary layer development. Further, it may be noted that the SST-V turbulence model solutions obtained for the full-geometry case with long $X_{t}$ and for the no-cone approach provide nearly identical boundary layer profiles. Considering that these two approaches match at the upstream station, comparison of the downstream wall surface quantities will yield effects of the far field differences between the full-geometry and no-cone approaches.

We turn next to comparison of wall surface quantities in the SWBLI focus region. Figure 7 compares the solutions for the differing modeling approaches. Note that the nondimensionalizing quantities $P_{W \infty}$ and $Q_{W \infty}$ are the wall pressure and surface heat transfer, respectively, at the $S=-6 \mathrm{~cm}$ station. Also, up to the origin, $S$ is measured axially along the cylinder surface, but then measured along the flare surface. The $S=0 \mathrm{~cm}$ station is the flare origin. While all of the solutions are relatively close to each other, it may be observed that there is an effect of the transition specification location. The short $X_{t}$ case, with transition point further downstream, has a smaller boundary layer entering the SWBLI region and a faster response in both wall pressures and surface heat transfer. The SST-V turbulence model solutions obtained for the full-geometry configuration with long $X_{t}$ and that with no cone are nearly identical through most of the 
SWBLI region, but differ near the end of the domain, and it is believed that this was due to the far field differences outside of the shock layer.

\section{Grid Sensitivity}

In this section, we consider grid effects for the 20-degree flare case. In line with the philosophy of the TMBWG and Turbulence Modeling Resource website, grid sensitivity investigations were conducted where a medium grid used every other point in each computational direction and a coarse grid used every 4th point in each direction. The specific modeling approach chosen here was for the full geometry with short $X_{t}$. The SST-V turbulence model was used. Figure 8 provides a comparison of velocity profiles and stagnation temperature profiles using these three grid levels. There appears to be minimal sensitivity to grid density at the upstream measurement station.

Grid sensitivities in the interaction region are presented in Fig. 9. While the solutions are very close, it cannot be concluded that grid sensitivity has been completely eliminated. However, an additional case was investigated where the wall spacing was halved relative to the baseline fine grid while the spacing near the oblique shock from the flare was kept the same, and resulted in a nearly identical solution to that of the fine grid presented in Fig. 9. It was concluded then that the discrepancy was due to imperfect capturing of the oblique shock. Unfortunately with a structured grid, it is not possible to perfectly tailor the grid to the shock as (1) the shock slightly curves at the beginning of the SWBLI region, and (2) the position of the shock moves slightly as a function of modeling approach and turbulence model selection. The fine grid as selected here, then, is considered as "grid independent" as may be practically achieved for this case, considering the aforementioned variations that would be expected with modeling variations. While not shown here, the SA model demonstrated the same trends with varying grid levels for the no-cone approach.

\section{Grid Orthogonality}

The grid sensitivity investigations used a baseline grid for the no-cone approach, as shown in Fig. 3, where the radial grid lines in the " $j$ " or direction normal to the test article surface were everywhere 90 degrees to the freestream flow direction, including along the flare which is oriented at 20 degrees. Along the surface of the flare, however, the flow within the shock layer would not be aligned with such a grid. The computational difficulty here is that the wall distance search routine in some structured grid codes (including Wind-US) is not exact; it finds the closest wall distance measured along grid lines. This approximate method is not strictly correct, and has been shown to lead to discrepancies with models that use the minimum distance. For example, the SA model includes a destruction term that is a function of the distance from the wall, and as shown in Ref. 16, can depend strongly on the method used to calculate the wall distance. The SST and BSL family of models uses the wall distance to compute switching functions to transition the model from the formulation used in the inner portion of the boundary layer to the formulation used in the outer portion, but has not shown the same sensitivity to grid topology for this particular case.

To examine this sensitivity for the 20-degree flare case, a new grid was constructed, also employing the no-cone approach, where all points in the near wall regions were orthogonal to the local test article surface. Upstream of the flare, this grid was identical to the baseline. Along the flare, however, the grid was transitioned immediately at the flare origin, such that the grid in the shock layer and containing all of the boundary layer was orthogonal to the flare surface. Figure 10 provides a comparison of solutions obtained with the initial baseline grid having radial grid lines to solutions obtained with a grid where the j-grid lines are orthogonal to the flare surface. The SA model produces a significant difference in the calculated heat flux depending on the grid used while the SST-V model shows negligible effect. The grid topology differences have minimal effect on calculated wall pressures. All of these solutions shown in Fig. 10 were performed with Wind-US, whose wall search method is along grid lines. When CFL3D was applied to the same two grids, but using a wall search method which found the actual closest wall distance, there were no differences between SA results obtained on the baseline and orthogonal grids.

\section{Turbulent Prandtl Number Effects}

For most aerodynamic calculations at transonic or lower speeds without significant heat transfer, the effect of turbulent Prandtl number is not usually very large as the mean flow is indirectly affected by the density variation. However, for high Mach number flows, and especially where heat transfer is important, the 
effect of $\mathrm{Pr}_{t}$ can be significant. ${ }^{17}$ For example, in the inner layer, the temperature profile can be shown to approximate ${ }^{18}$

$$
T=T_{w}-\frac{P r_{t}}{C_{p}} \frac{Q_{w}}{\tau_{w}} u-\frac{P r_{t} u^{2}}{2 C_{p}}
$$

DNS analysis of the compressible law of the wall has implied $P r_{t}$ is approximately 0.9 near the log layer $^{19}$ and this value is in accordance with the incompressible case. ${ }^{20}$ Unfortunately, assuming constant $\mathrm{Pr}_{t}$ in a prediction method cannot be justified. There seems to have some inconsistency between DNS and experiments as regards to the range of $P r_{t}$. The experimental data indicate $P r_{t}$ increases to around 2 near the wall ${ }^{21}$ while the DNS indicates an asymptotic value of 1.1 near the wall. In the freestream $P r_{t}$ falls to a value of approximately 0.5 . In the study, we therefore consider large variations in constant values for $P r_{t}$, with variation from $0.5 \leq P r_{t} \leq 2.0$ as suggested in the range of the previous observation.

Figure 11 compares inflow station profiles obtained with SST-V when varying $P r_{t}$. As expected, there appears to be minimal effect on the velocity profiles, but significant effect on the density and stagnation temperature profiles. As the heat transfer rate, $Q_{w}$, is negative, Eqn. 8 implies that the temperature will display a peak near the inner layer region. The fact that the solution with $\operatorname{Pr}_{t}=0.9$ matches the peak profile better indicates that an assumption of $\operatorname{Pr}_{t}=0.9$ for the inner layer may be adequate. Table 2 compares integral thicknesses for the three turbulent Prandtl numbers. In agreement with the velocity profile comparison, there is only a small effect on the velocity thickness but more substantial effect on the displacement and momentum thicknesses. None of the cases enable good agreement with the experimentally measured momentum thickness. Figure 12 provides a comparison of wall quantities obtained with SST-V when varying $\mathrm{Pr}_{t}$. There is more variation in the wall heat transfer than wall surface pressure, as may be expected, however, the $P r_{t}=2.0$ case may be observed to have a separation pushed further forward of the leading edge than for the lower $P r_{t}$ cases. This is because a higher value of $P r_{t}$ leads to a higher peak temperature profile (and hence a lower density profile) in the inner boundary layer. As a consequence, there is less momentum transport in the inner layer for higher value of $\operatorname{Pr}_{t}$ (because of lower density) and hence the flow is prompt to separation, as seen in Fig. 12.

Table 2. Comparison of inflow station $\left(S=-6 \mathrm{~cm}\right.$ boundary layer state.), $\operatorname{Pr}_{t}$ effects.

\begin{tabular}{lccc}
\hline Case & $\delta_{v}(\mathrm{~cm})$ & $\delta^{*}(\mathrm{~cm})$ & $\theta(\mathrm{cm})$ \\
\hline \hline Experimental Data & 0.207 & 0.735 & 0.0642 \\
SST-V, No Cone, $\operatorname{Pr}_{t}=0.5$ & 0.226 & 0.842 & 0.0519 \\
SST-V, No Cone, $\operatorname{Pr}_{t}=0.9$ & 0.219 & 0.762 & 0.0502 \\
SST-V, No Cone, $\operatorname{Pr}_{t}=2.0$ & 0.215 & 0.680 & 0.0478 \\
\hline
\end{tabular}

\section{Code Effects}

Most cases presented on the previously mentioned Turbulence Modeling Resource website have been validated with at least two different RANS solvers. For the cases considered here, CFL3D and Wind-US were employed. The benefit of using more than one code was demonstrated here with the disagreement in SA results and subsequent discovery of Wind-US dependence on grid orthogonality in its minimum distance function. The results presented in this section use the grid everywhere orthogonal to the test article and the no-cone inflow modeling approach.

Figure 13 compares inflow station profiles obtained with both codes and the SA and SST-V models. It may be concluded that the results are relatively code-independent, although the SA results show some minor differences in the density profiles at the outer portion of the boundary layer. An SST result obtained with CFL3D (again recall this form of the model uses the rate of strain in the production term in contrast to SST-V which uses vorticity) is also presented which shows essentially no differences from the SST-V results of either code.

Examining Fig. 14, the code-to-code comparisons of SA and SST-V, respectively, show no major differences for wall surface quantities. However, the SST result for wall heat transfer may be observed to be quite 
different from SST-V. This finding only came from obtaining a large difference in solutions that the authors initially thought were both SST results, and not initially suspecting that the underlying production term formulation would produce such a difference. Only after obtaining a true SST-V solution with both codes and SST solution with CFL3D did the cause of the difference become clear. Figure 15 provides a comparison of eddy viscosity contours obtained with SST and SST-V in CFL3D. While Ref. 15 discusses motivation for the usage of vorticity-based production in stagnation point problems, the current result demonstrates the significant differences between rate-of-strain magnitude and vorticity magnitude where the flow turns due to the compression corner.

\section{Turbulence Modeling Effects}

While some of the earlier sections of this report considered multiple turbulence models, the focus of this last section for the 20-degree flare case is variations in the turbulence models, while holding everything else fixed. The orthogonal fine grid, with $P r_{t}=0.9$, and no-cone inflow approach is used here. The inflow profiles are not presented as there is virtually no difference between any of the $k-\omega$ model results at the upstream location. That is, SST, SST-V, SST-V with modified shear stress limiter $\left(a_{1}\right)$, and BSL-V yield nearly identical inflow profiles at the upstream measurement station.

Wall surface quantities are presented in Fig. 16 and compare SA with four Menter $k-\omega$ model variations. The SA solution and the three vorticity based production term cases (SST-V, SST-V with modified shear stress limiter, and BSL-V) were obtained with Wind-US while the SST model solution that employs the strain-rate production term was obtained with CFL3D. The previous section demonstrated that if the model formulation was truly identical, the two codes would provide nearly identical results.

Figure 16 shows that while there is not substantial variation in wall pressures, there is notable variation in the predicted heat flux. The BSL-V variant of the Menter turbulence model, which differs from SST-V in that there is no limit imposed on the turbulent shear stress, produces a solution that differs substantially. Just as large a variation, however, is also observed between the SST and SST-V solutions, demonstrating how significant an effect that the choice of production term approximation has on the resultant SWBLI predictions. The SA and SST-V solutions agree best with the experimental data for the wall heat flux for this case.

\section{0-Degree Flare Results}

In this section, we consider a more challenging flow case where the flare angle is increased from 20 degrees to 30 degrees. The Mach number contours in Fig. 17, obtained with the SST-V model and no-cone approach, show that the steeper compression corner results in a much larger flow deficit region, compared with that for the 20-degree flare (see Fig. 5). All of the parametrics considered for the 20-degree case were not considered for the 30-degree case. For all of the solutions considered in this section, an orthogonal fine grid, with $\operatorname{Pr}_{t}=0.9$, and no-cone inflow approach was used. Further, the grid upstream of the interaction was identical, and none of the solutions had the interaction pushing forward of the inflow measurement plane at $S=-6 \mathrm{~cm}$, so that the inflow profiles for this case were identical to those of the 20-degree flare, with all of the modeling settings the same. The SST and SST-V solutions did have the interaction region push close to but not all the way up to the $S=-6 \mathrm{~cm}$ location.

\section{Grid and Code Effects}

Figure 18 provides a comparison of solutions obtained for the 30-degree cone case, showing grid density variations. It may be observed that the differences between medium and fine level grids are minor, and on the same order of magnitude as for the 20-degree flare case. The SST-V grid resolution variations were obtained with Wind-US while the SST variations were obtained with CFL3D. An additional solution shown in this figure, from CFL3D with SST-V, may be compared with the Wind-US solution and indicates very close agreement. Interestingly, the SST versus SST-V differences are, on a relative basis, not as large for this more challenging flow than for the less challenging 20-degree case, which is because the separation spreads out the pressure gradient. 


\section{Turbulence Modeling Effects}

Figure 19 provides a comparison of solutions obtained with the different turbulence models for the 30-degree flare case, presented in an analogous manner to the 20-degree flare case shown in Fig. 16. Here, the variation due to the shear stress limiter is much more apparent with the larger flow reversal region. Unlike the 20degree case, there is substantial difference in the pressure profiles. The SST and SST-V solutions indicate that the interaction has reached approximately $S=-6 \mathrm{~cm}$, while the BSL and SA solutions indicate minimal SWBLI migration upstream and agree more closely with data. There is even greater variation in the wall heat transfer results. Interestingly, the trend in the heat transfer predictions moving from BSL-V to SST-V is opposite that of the 20-degree case. This is due to the much stronger interaction for the 30-degree case, and very large separation produced by the SST-V solution. The SST-V solution with modified $a_{1}$ limiter falls between the SST-V and BSL-V solutions. The SST and SST-V solutions provide the greatest overshoot in peak heat transfer rate. The SA heat transfer reaches a lower peak than any of the two-equation model results. The SA and BSL-V solutions agree best with the experimental data for the wall wear flux for this case.

\section{Conclusions}

Two shock-wave / boundary-layer interaction (SWBLI) cases, from the axisymmetric compression corner experiments of Kussoy and Horstman, were investigated using one- and two-equation Reynolds-averaged Navier-Stokes (RANS) turbulence models. These cases were investigated previously in other published efforts, but were revisited in this work, in an effort by the AIAA Turbulence Modeling Benchmarking Working Group to extend test cases to higher Mach numbers beyond the current repository of the NASA Turbulence Modeling Resource website.

The two test cases were of relatively simple geometry, having axisymmetric compression corner geometries with SWBLI inducing flares of 20 and 30 degrees, respectively. The freestream Mach number was approximately 7. The RANS closures examined were the Spalart-Allmaras (SA) one-equation model and several variants of the Menter family of $k-\omega$ two-equation models. Most of the modeling parametrics were conducted for the 20-degree flare. Beyond strictly turbulence model effects, inflow modeling strategy, grid resolution, grid orthogonality, turbulent Prandtl number, and code-to-code variations were also considered.

An overall domain that replicated the experimental geometry from the leading edge of the test article was compared with a second approach that did not replicate all of the upstream details but had a straight inflow section constructed to match the boundary layer integral thicknesses measured in the experiments. While the comparisons of solutions obtained with these two approaches revealed some differences, the simplified geometry referred to as the "no-cone" approach was deemed to be sufficient.

Grid resolution studies showed that a nearly grid independent answer could be obtained for a mesh with 401 grid points normal to the test article surface and 321 points axially placed between the inflow measurement station and the outflow plane. For these cases with strong oblique shocks at high Mach numbers, grid packing to the wall was of course necessary, but some grid tailoring to the oblique shock was also found to be necessary. Because the flow solutions changed somewhat with turbulence model selection, a single computational grid tailored for one model was not perfectly tailored to another. Using a grid not orthogonal to the wall was found to have a significant effect on structured grid solutions for the SA model when using an inexact wall search routine that traveled along constant radial grid lines.

The turbulent Prandtl number, $P r_{t}$ was found to have a substantial effect on the inflow density and temperature profiles as well as downstream wall heat transfer. However, no single fixed value for $P r_{t}$ can be recommended as the preferred setting for either of the flow cases under consideration or other similar high Mach number flows.

When holding all of these other modeling variations fixed, the comparison of SA results to the four $k-\omega$ model results yielded some variation in surface static pressures, but more substantial variation in wall heat transfer. The differences between turbulence models was greater for the 30-degree flare case than for the 20-degree case, which is in agreement with previously referenced studies of the same test configuration. Considering both wall pressures and heat flux, the SA and SST-V models agreed best with experiment for the 20-degree case, and the SA and BSL-V agreed best with experiment for the 30-degree case. However, no model matched well everywhere. 


\section{References}

2010.

${ }^{1}$ Benek, J., "Overview of the 2010 AIAA Shock Boundary Layer Interaction Workshop," AIAA Paper 2010-4821, January

${ }^{2}$ Benek, J. and Babinsky, H., "Lessons Learned from the 2010 AIAA Shock Boundary Layer Interaction Workshop," AIAA Paper 2010-4825, January 2010.

${ }^{3}$ DeBonis, J. R., Oberkampf, W. L., Wolf, R. T., Orkwis, P. D., Turner, M. G., Babinsky, H., and Benek, J. A., "Assessment of Computational Fluid Dynamics and Experimental Data for Shock Boundary-Layer Interactions," AIAA Journal, Vol. 50, No. 4, 2012, pp. 891-903.

${ }^{4}$ Rumsey, C., Smith, B., and Huang, G., "Description of a Website Resource for Turbulence Modeling Verification and Validation," AIAA Paper 2010-4742, June 2010.

${ }^{5}$ Kussoy, M. I. and Horstman, C. C., "Documentation of Two- and Three-Dimensional Hypersonic Shock Wave Boundary Layer Interaction Flows," NASA TM 101075, January 1989. 1993.

${ }^{6}$ Huang, P. G. and Coakley, T. J., "Turbulence Modeling for Complex Hypersonic Flows," AIAA Paper 93-0200, January

${ }^{7}$ Olsen, M. E., Lilliard, R. P., and Coakley, T. J., "The Lag Model Applied to High Speed Flows," NAS Technical Report 05-005, May 2005.

${ }^{8}$ Nelson, C., "An Overview of the NPARC Alliance's Wind-US Flow Solver," AIAA Paper 2010-27, January 2010.

${ }^{9}$ Georgiadis, N. J., Yoder, D. A., Vyas, M. A., and Engblom, W. A., "Status of Turbulence Modeling for Hypersonic Propulsion Flowpaths," AIAA Paper 2011-5917, July 2011.

${ }^{10}$ Krist, S. L., Bierdon, R. T., and Rumsey, C. L., "CFL3D User's Manual (Version 5.0)," NASA TM 1998-208444, June 1998.

${ }^{11}$ Menter, F. R., "Two Equation Eddy-Viscosity Turbulence Models for Engineering Applications," AIAA Journal, Vol. 32, No. 8, 1994, pp. 1598-1605.

${ }^{12}$ Spalart, P. R. and Allmaras, S. R., "A One-Equation Turbulence Model for Aerodynamic Flows," AIAA Paper 92-0439, 1992.

${ }^{13}$ Spalart, P. R. and Allmaras, S. R., "A One-Equation Turbulence Model for Aerodynamic Flows," La Recherche Aerospatiale, , No. 1, 1994, pp. 5-21.

${ }^{14}$ Georgiadis, N. J. and Yoder, D. A., "Recalibration of the Shear Stress Transport Model to Improve Calculation of Shock Separated Flows," AIAA Paper 2013-0685, January 2013.

${ }^{15}$ Menter, F. R., "Improved Two Equation $k-\omega$ Turbulence Models for Aerodynamic Flows," NASA TM 103975, October 1992.

${ }^{16}$ Levy, D. W., Laflin, K. R., Tinoco, E. N., Vassberg, J. C., Mani, M., Rider, B., Rumsey, C. L., Wahls, R. A., Morrison, J. H., Broderson, O. P., Crippa, S., Mavriplis, D. J., and Murayama, M., "Summary of Data from the Fifth Computational Fluid Dynamics Drag Prediction Workshop," Journal of Aircraft, Vol. 51, No. 4, 2014, pp. 1194-1213.

${ }^{17}$ Georgiadis, N. J., Yoder, D. A., Vyas, M. A., and Engblom, W. A., "Status of Turbulence Modeling for Hypersonic Propulsion Flowpaths," Theor. Comput. Fluid Dyn., Vol. 28, 2014, pp. 295-318.

${ }^{18}$ Huang, P. G., Bradshaw, P., and Coakley, T. J., "Skin Friction and Velocity Profile Family for Compressible Turbulent Boundary Layers," AIAA Journal, Vol. 31, No. 9, 1993, pp. 1600 - 1604.

${ }^{19}$ Huang, P. G., Coleman, G. N., and Bradshaw, P., "Compressible turbulent channel flows - DNS results and Modeling," Journal of Fluid Mechanics, Vol. 305, 1995, pp. 185-218.

${ }^{20}$ Bradshaw, P. and Huang, P. G., "The Law of the Wall in Turbulent Flows," Proc. Roy. Soc. Lond. A, Vol. 451, 1995, pp. 165-188.

${ }^{21}$ Kays, W. M., "Turbulent Prandtl Number - Where Are We?" Transactions of the ASME, Journal of Heat Transfer, Vol. 116, May 1994, pp. 284-295. 


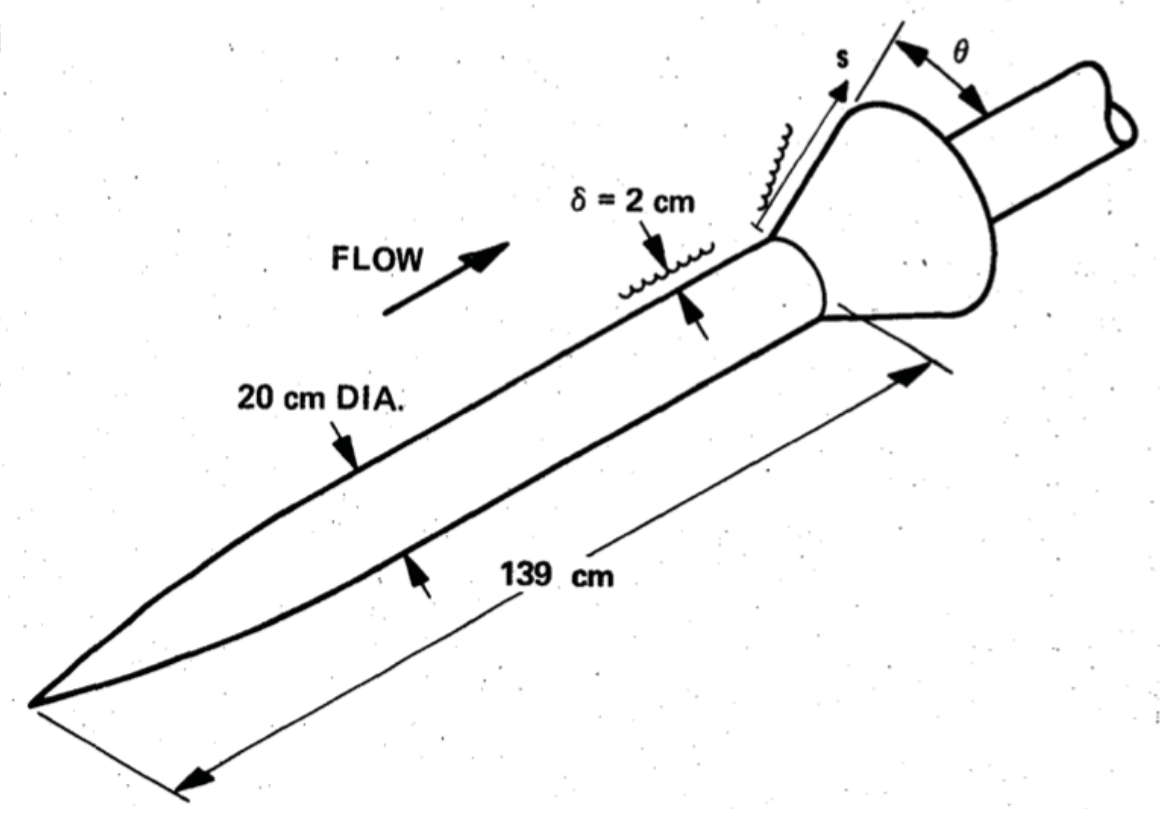

Figure 1. Test article geometry for axisymmetric compression corner flow of Kussoy and Horstman (taken from Ref. 5.) 


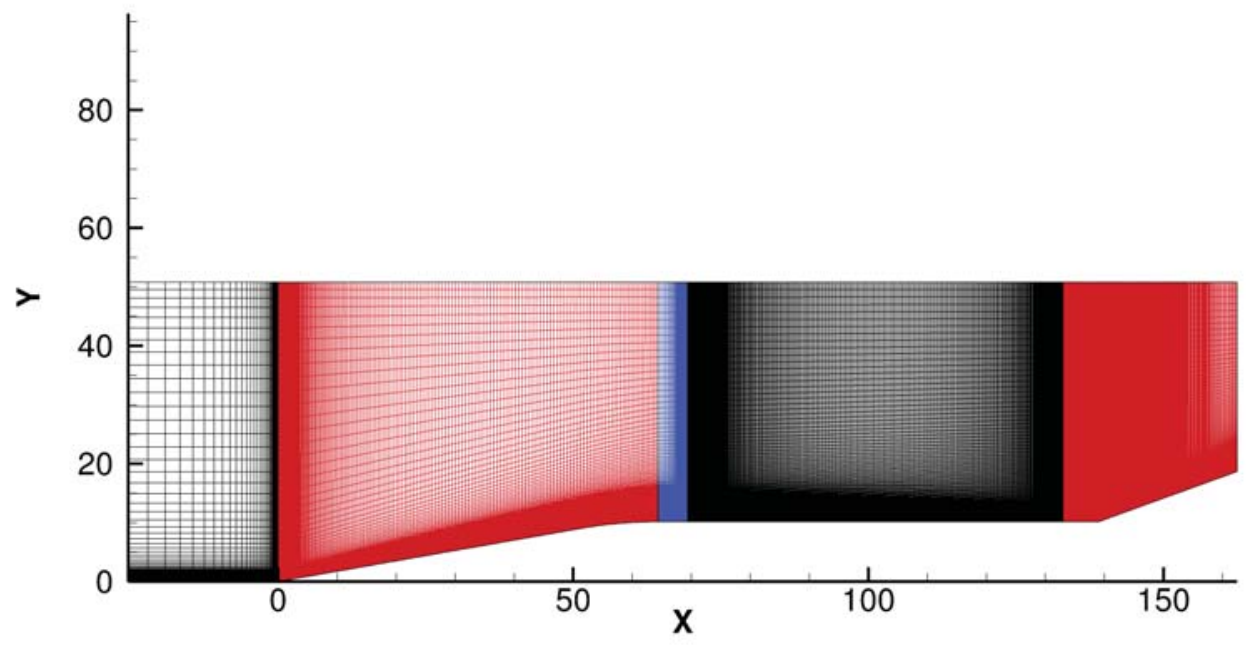

(a) Full Geometry

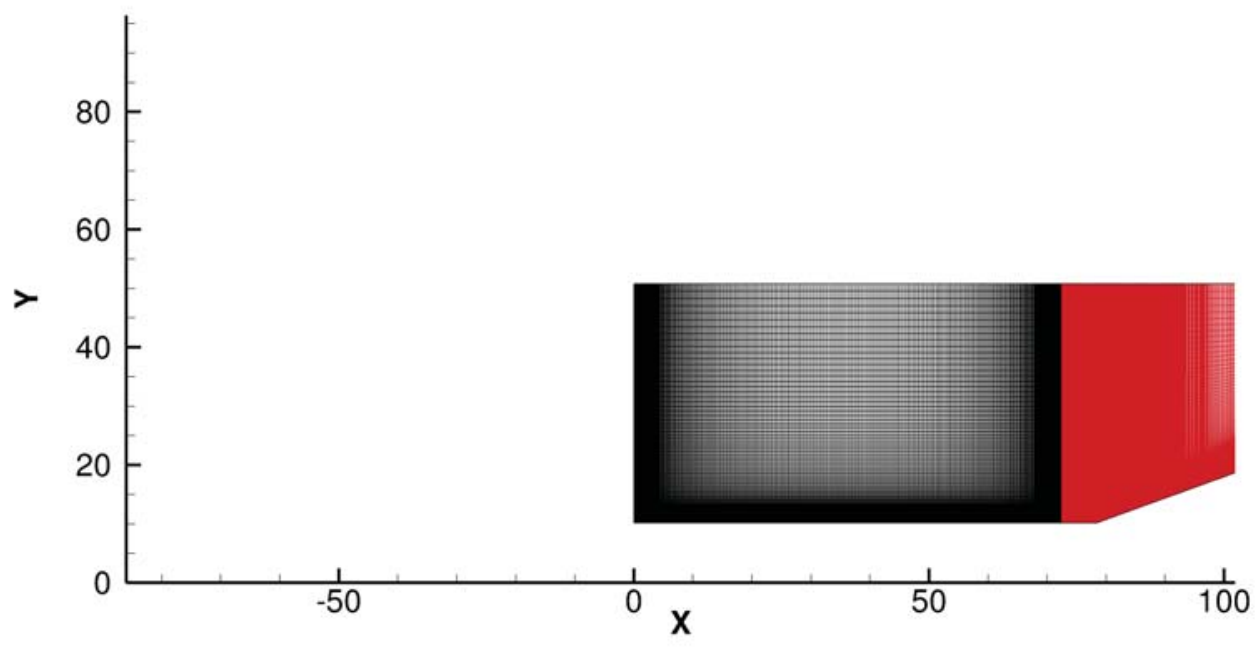

(b) No Cone

Figure 2. Computational grids illustrating the two approaches to overall modeling strategy. 


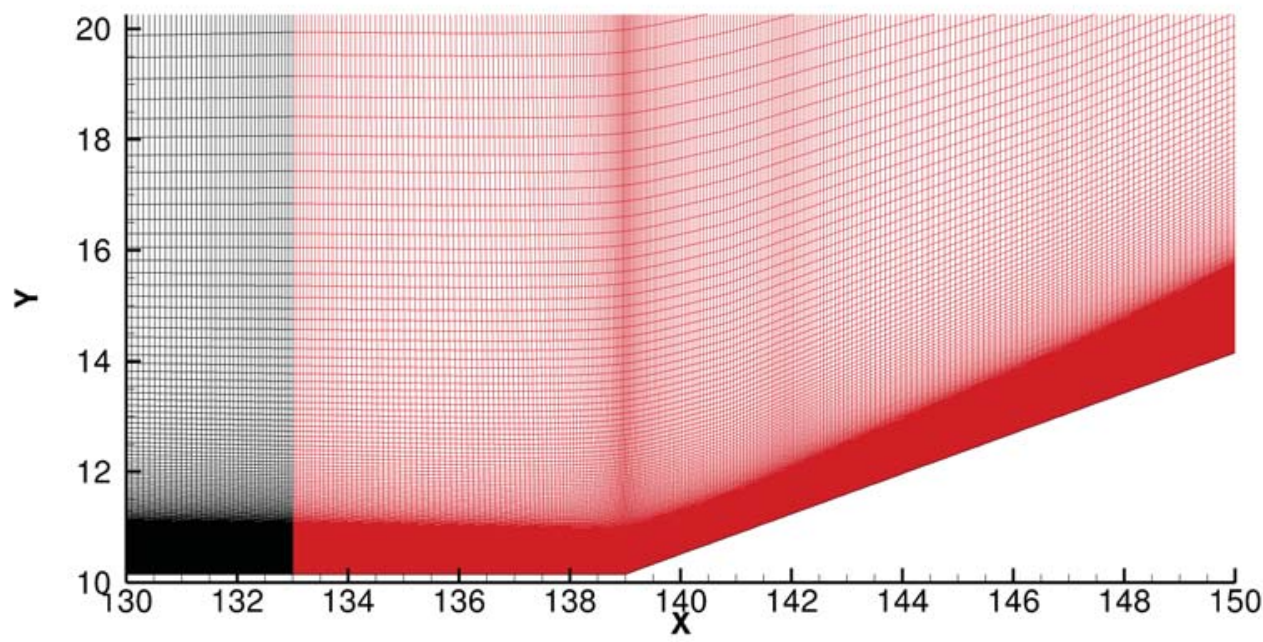

Figure 3. Closeup of grid (both approaches) near SWBLI focus region. 


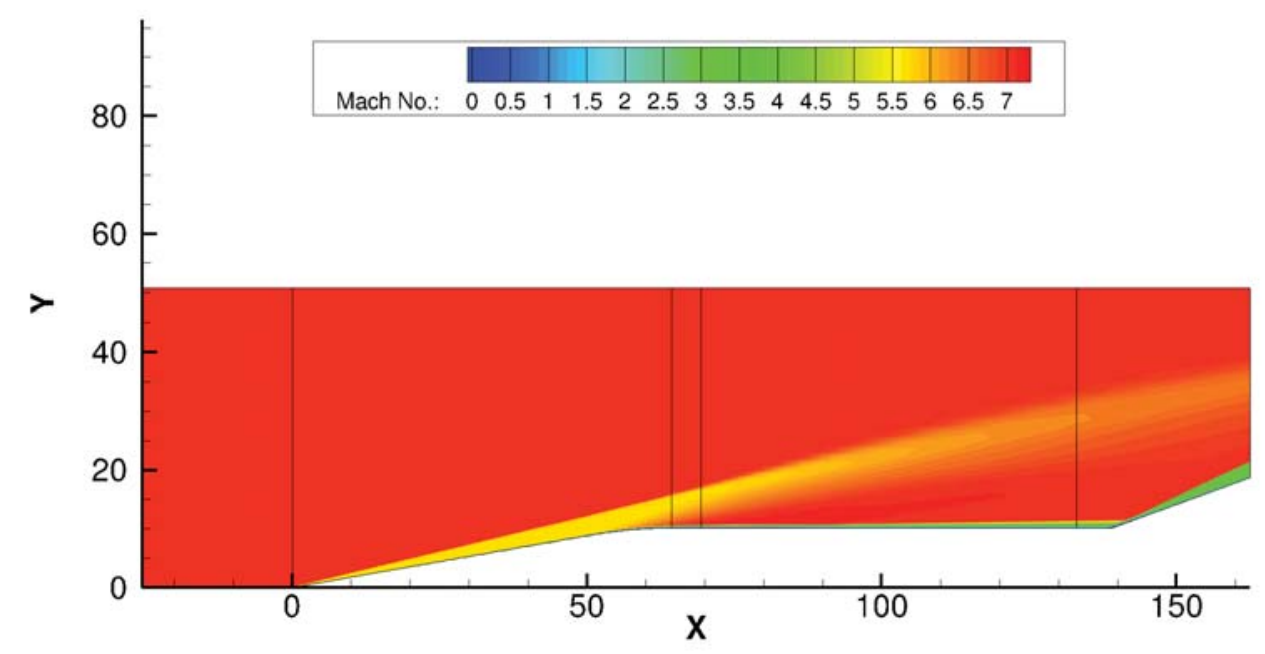

(a) Full Geometry

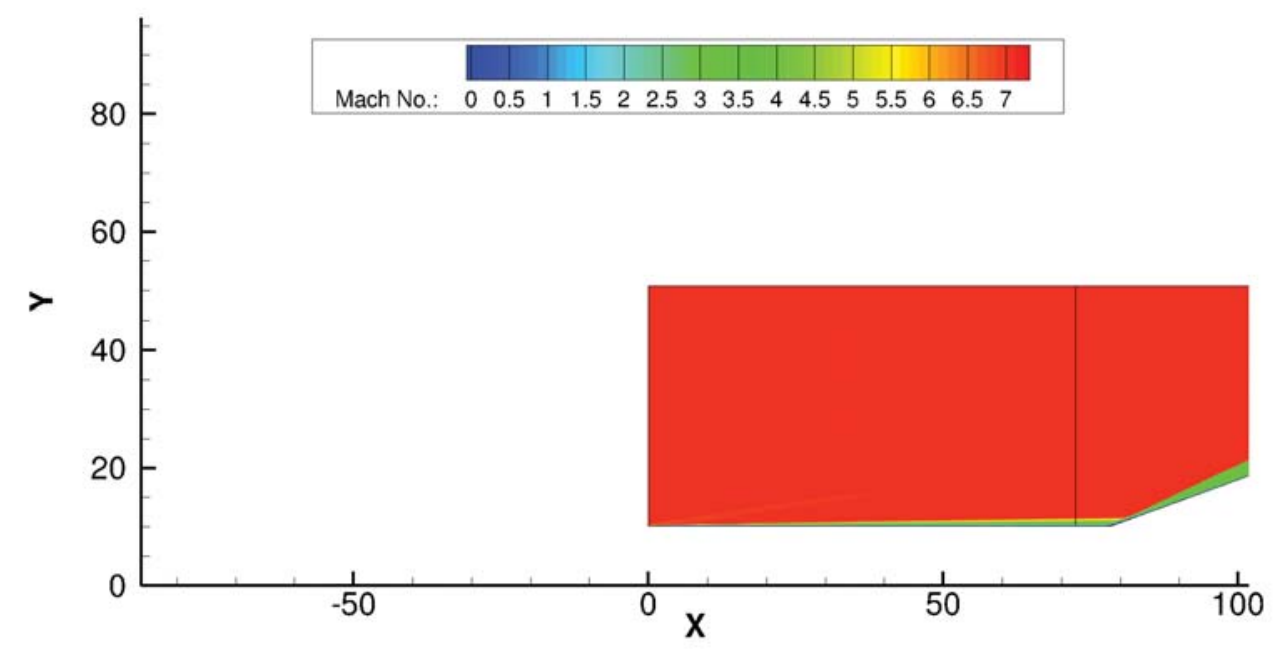

(b) No Cone

Figure 4. Mach number contours for the two approaches. 


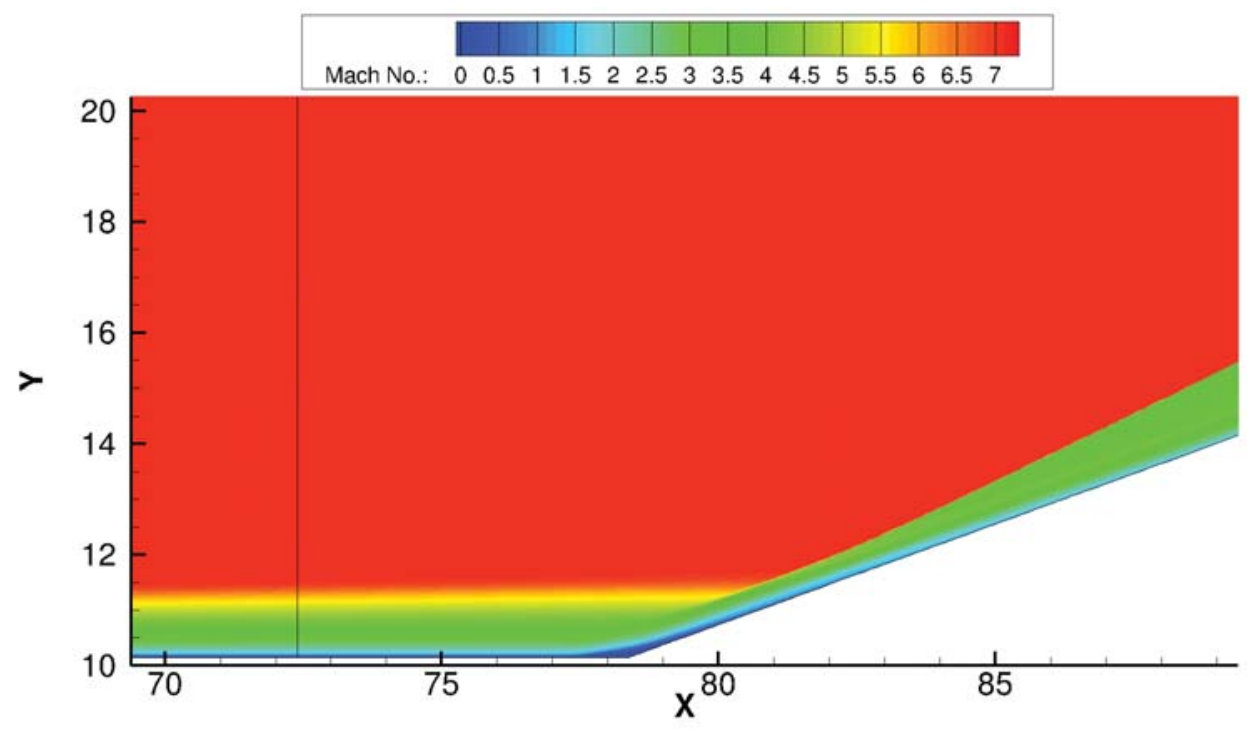

Figure 5. Closeup of Mach number contours (no-cone approach) near SWBLI focus region for 20-degree flare.

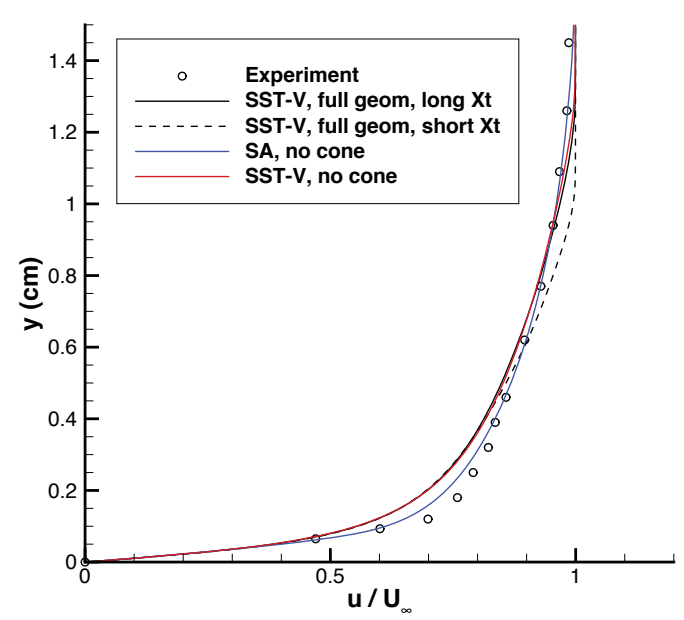

(a) Axial Velocity

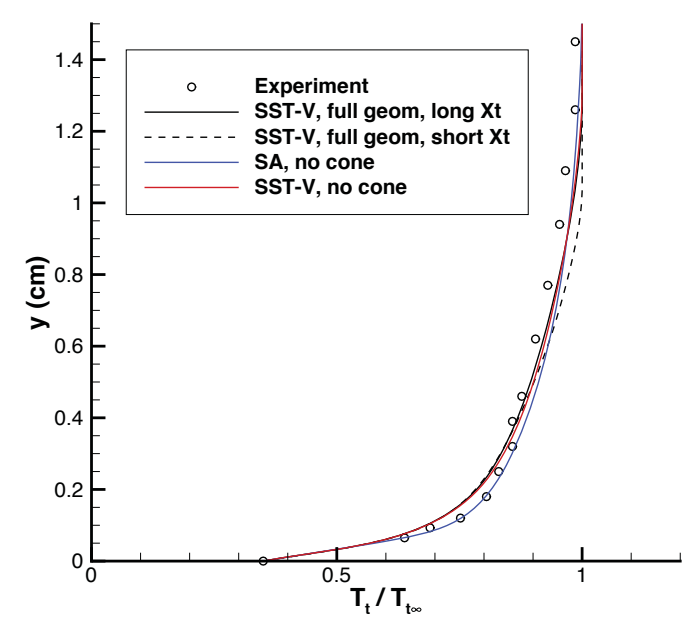

(b) Stagnation Temperature

Figure 6. Inflow boundary layer profile comparisons, different modeling approaches. 


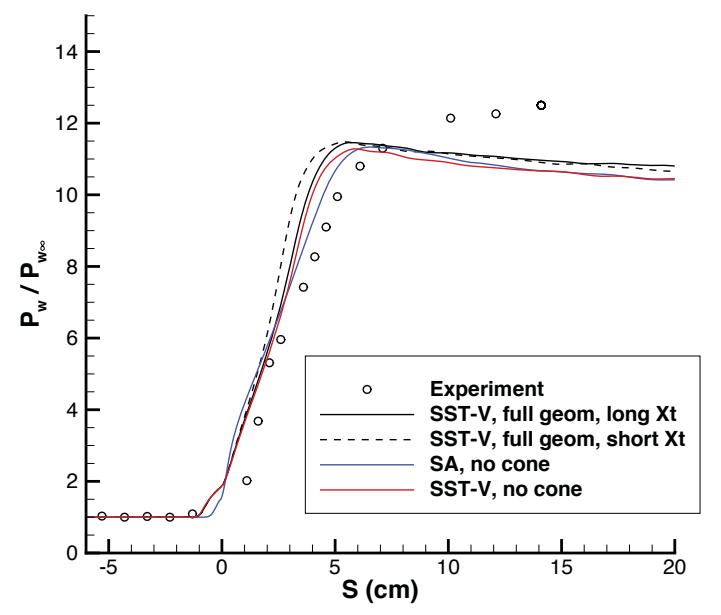

(a) Wall Pressures

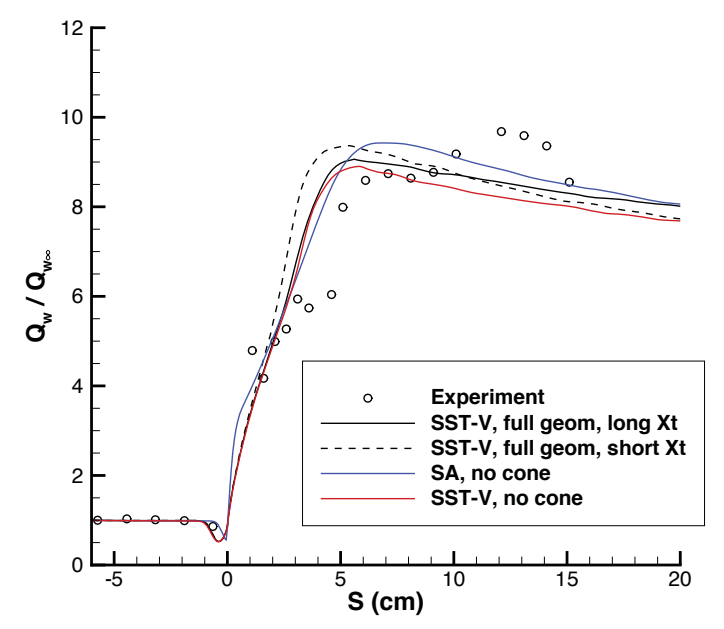

(b) Wall Heat Transfer

Figure 7. Comparison of surface quantities through SWBLI region, different modeling approaches.

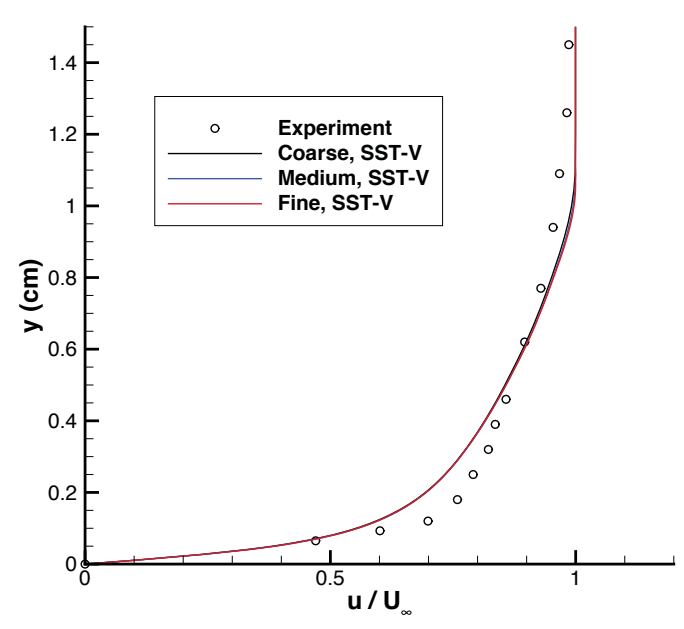

(a) Axial Velocity

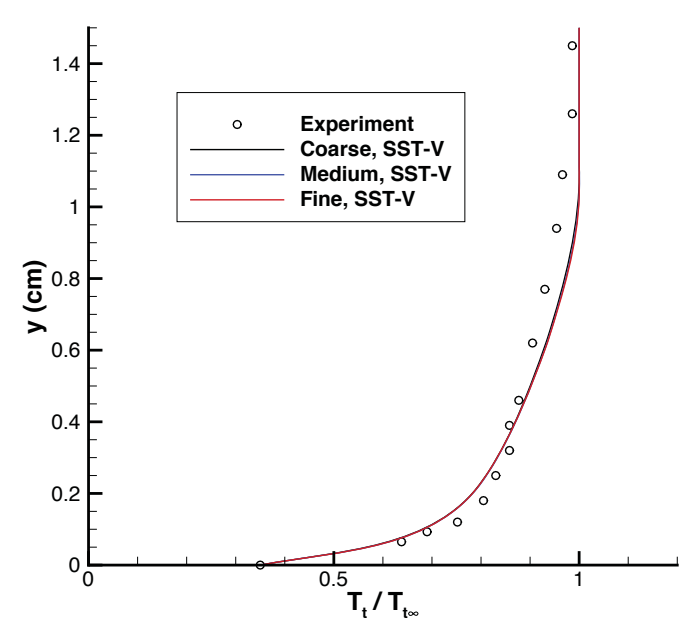

(b) Stagnation Temperature

Figure 8. Inflow boundary layer profile comparisons, at consecutive grid levels. 


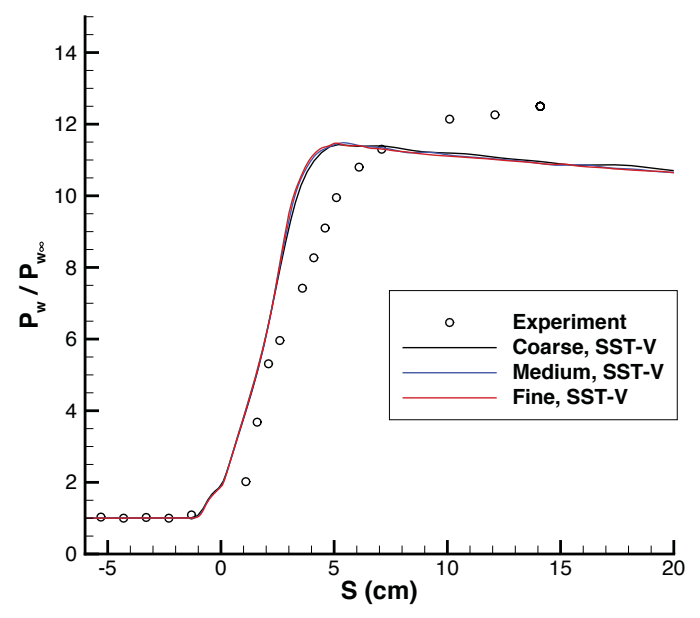

(a) Wall Pressures

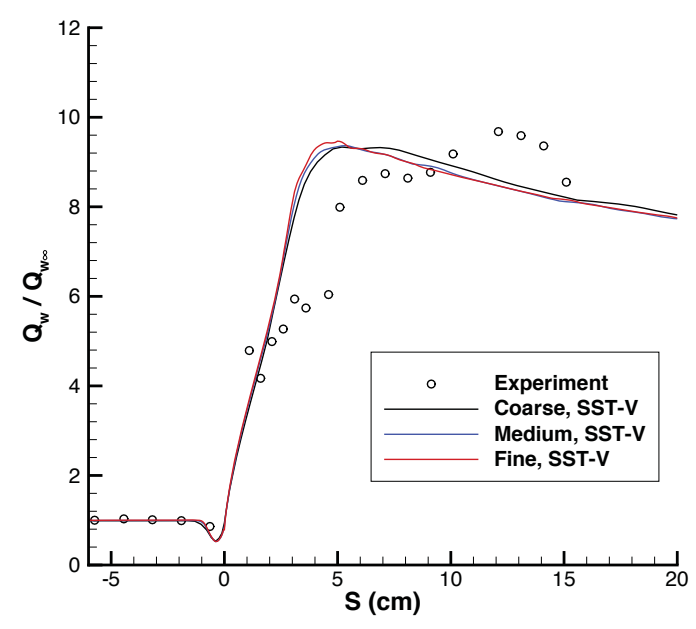

(b) Wall Heat Transfer

Figure 9. Comparison of surface quantities through SWBLI region, grid effects.

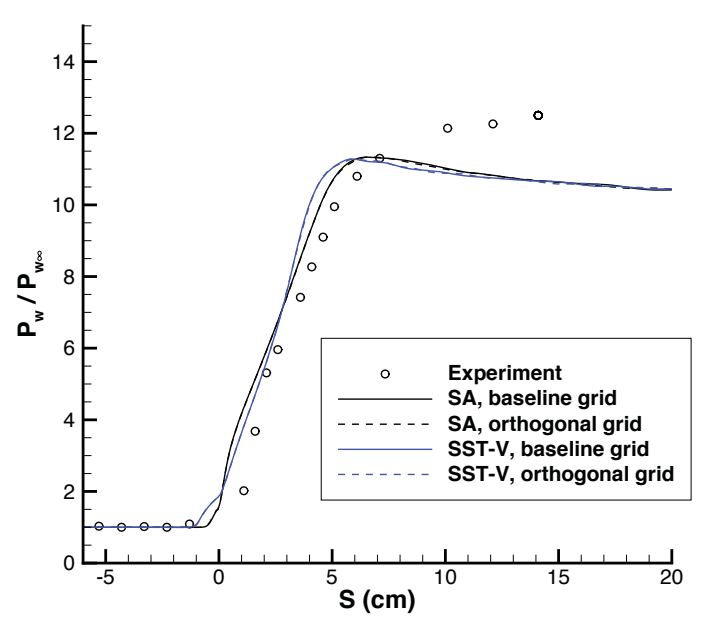

(a) Wall Pressures

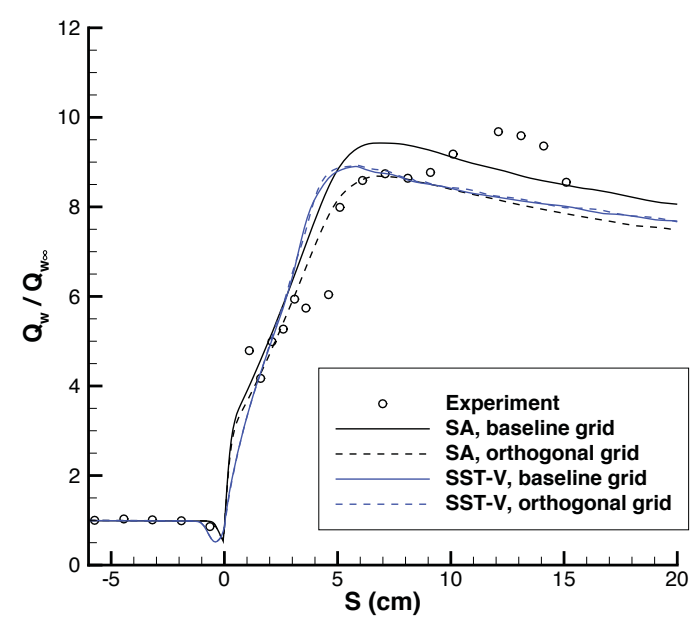

(b) Wall Heat Transfer

Figure 10. Comparison of surface quantities through SWBLI region, grid topology effects. 


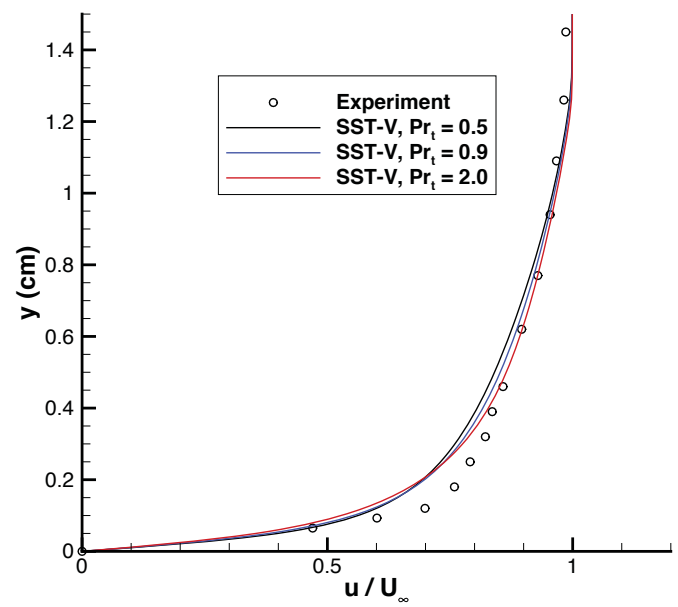

(a) Axial Velocity

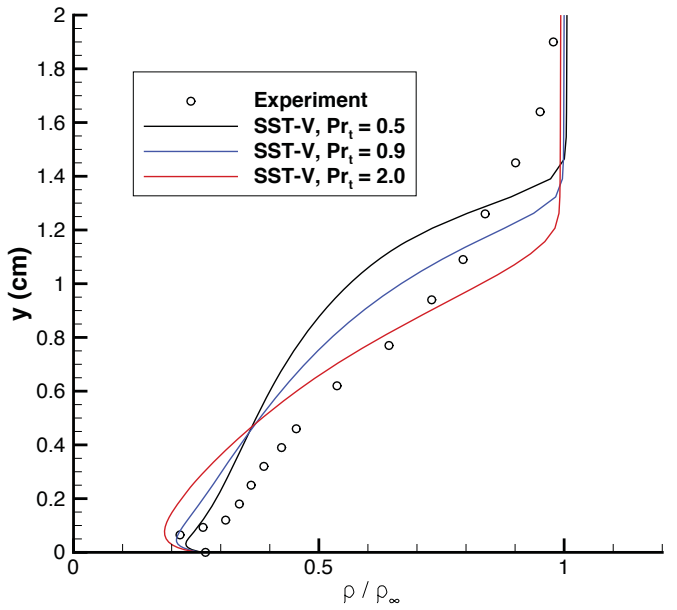

(b) Density

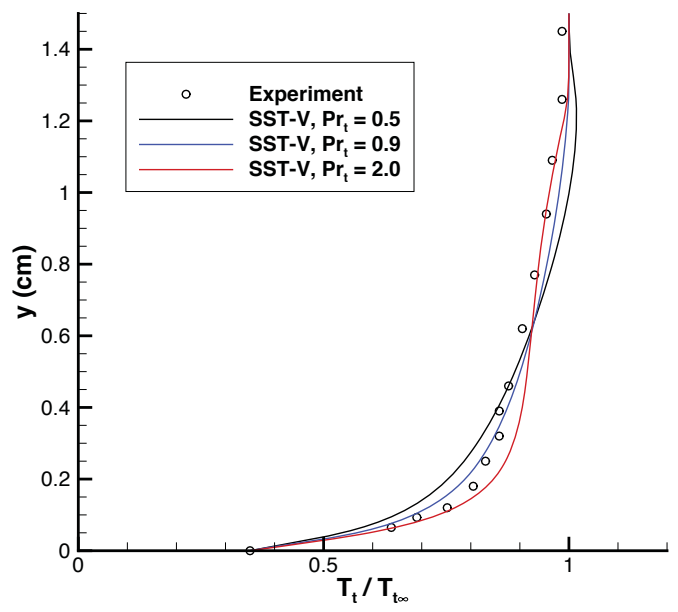

(c) Stagnation Temperature

Figure 11. Inflow boundary layer profile comparisons, $P r_{t}$ effects. 


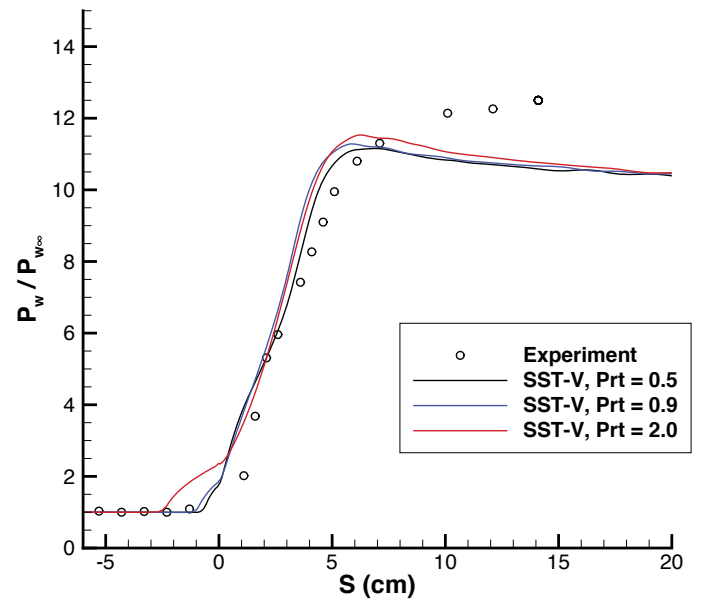

(a) Wall Pressures

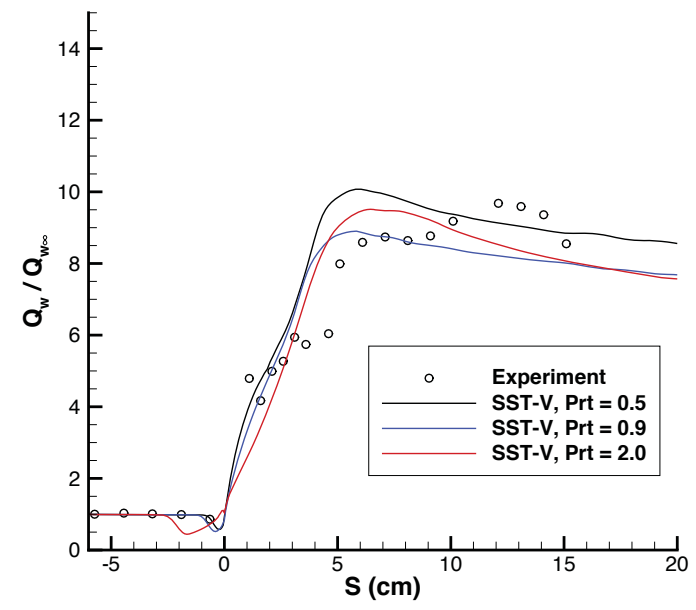

(b) Wall Heat Transfer

Figure 12. Comparison of surface quantities through SWBLI region, $P r_{t}$ effects. 


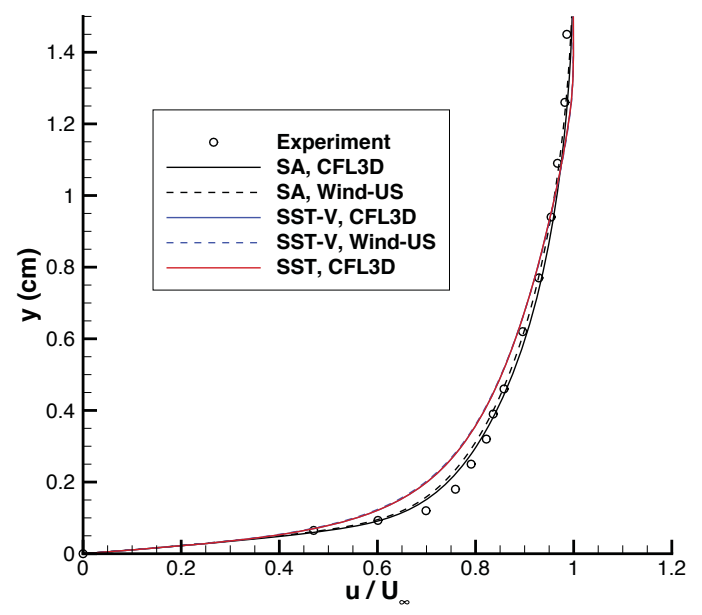

(a) Axial Velocity

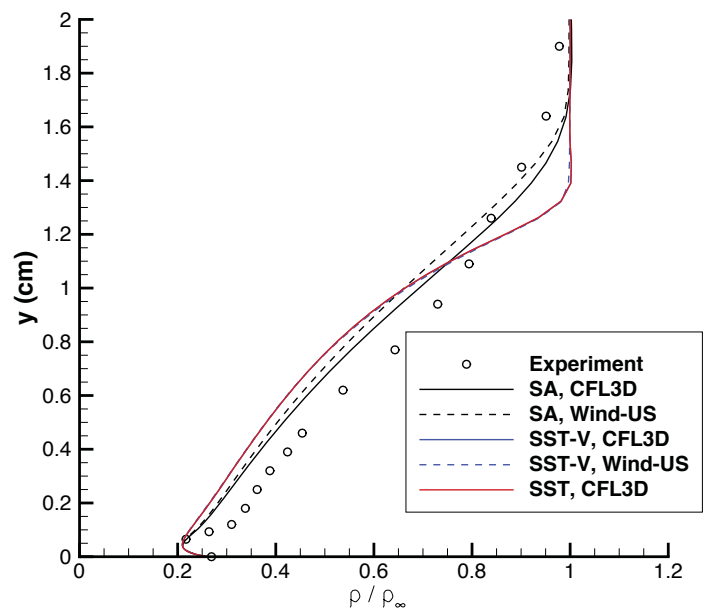

(b) Density

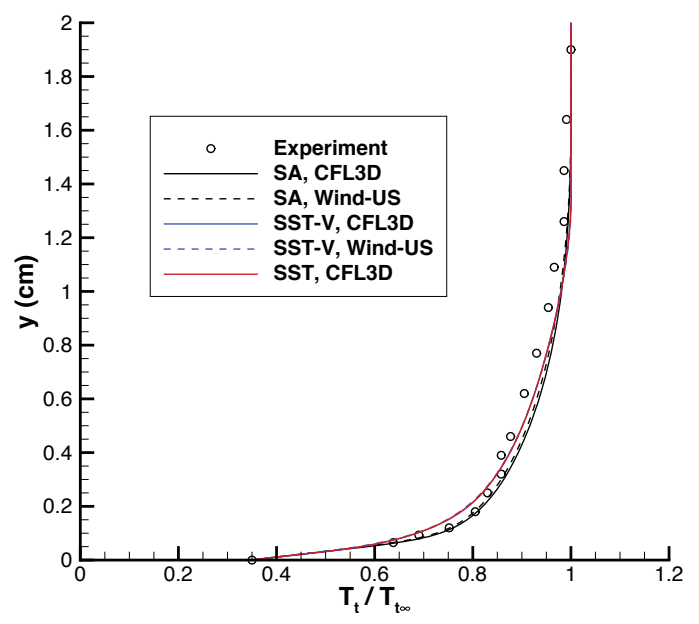

(c) Stagnation Temperature

Figure 13. Inflow boundary layer profiles 


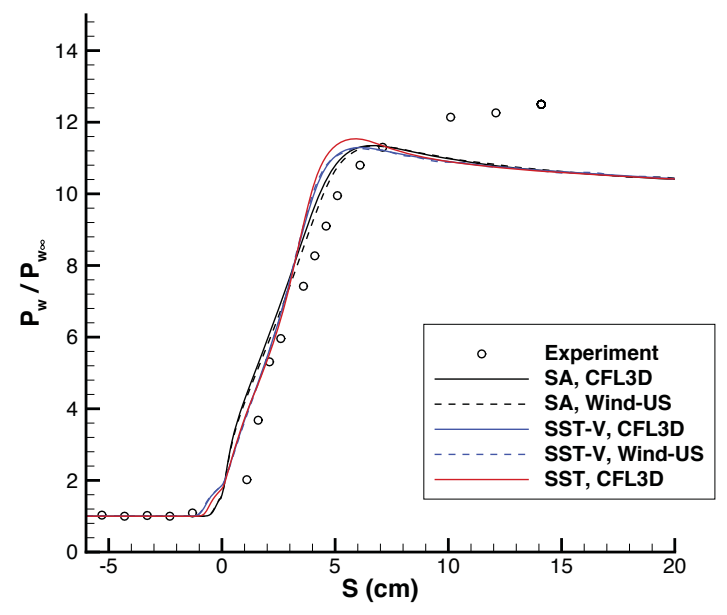

(a) Wall Pressures

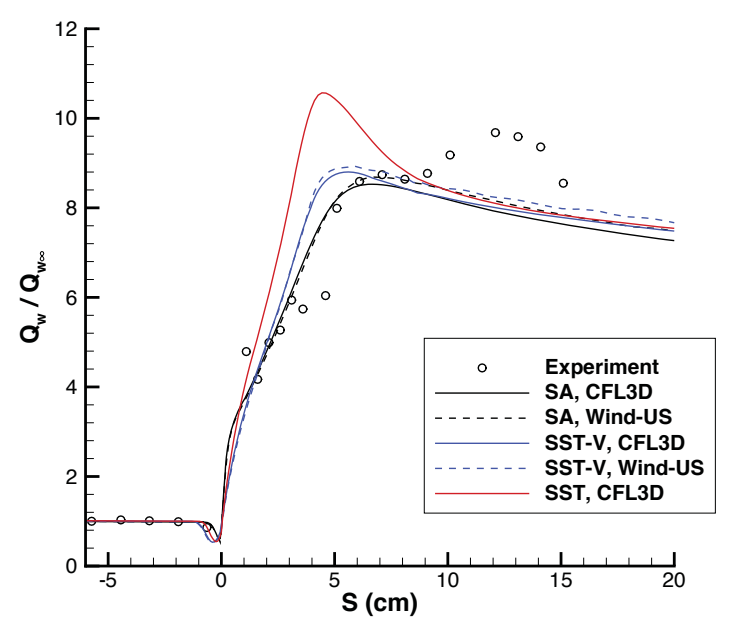

(b) Wall Heat Transfer

Figure 14. Comparison of surface quantities through SWBLI region, code effects. 


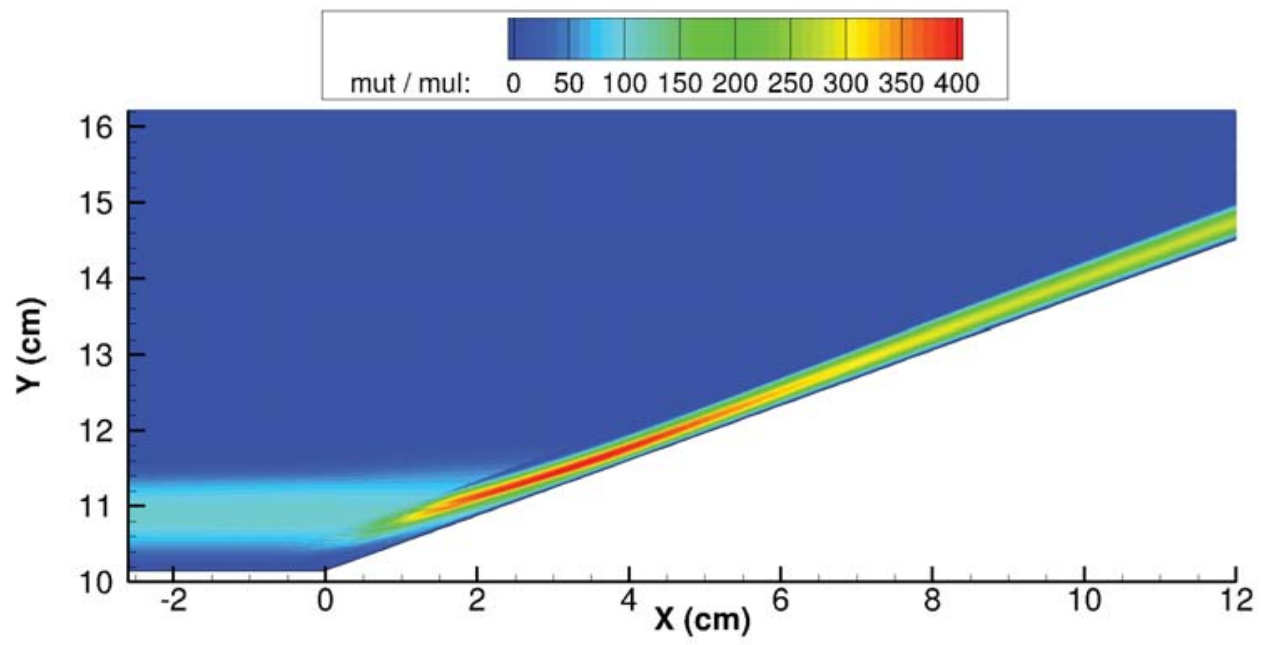

(a) $\mathrm{SST}$

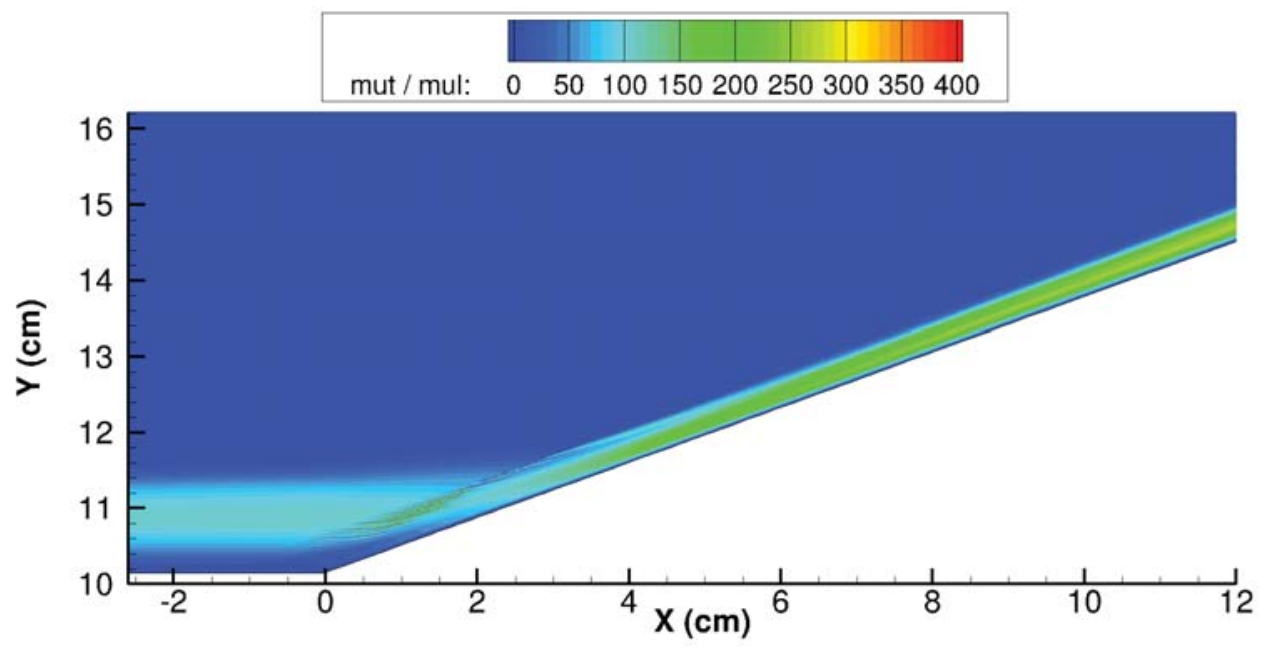

(b) SST-V

Figure 15. Eddy viscosity contours near origin of the flare. 


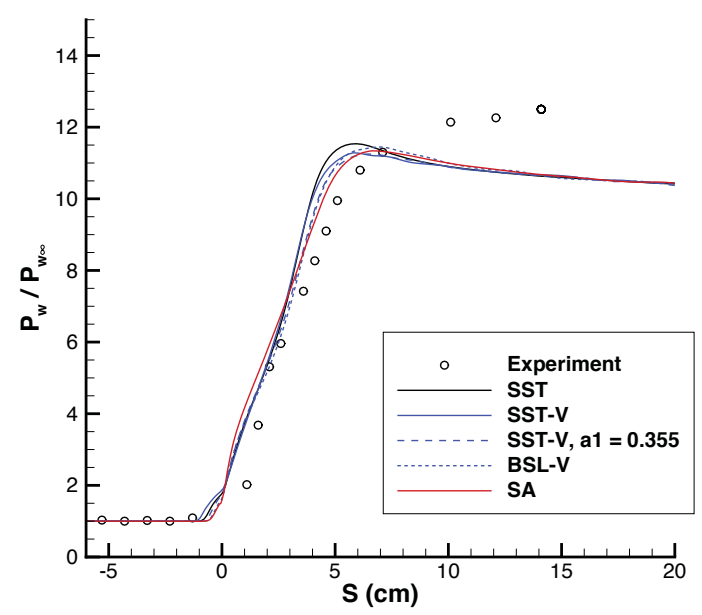

(a) Wall Pressures

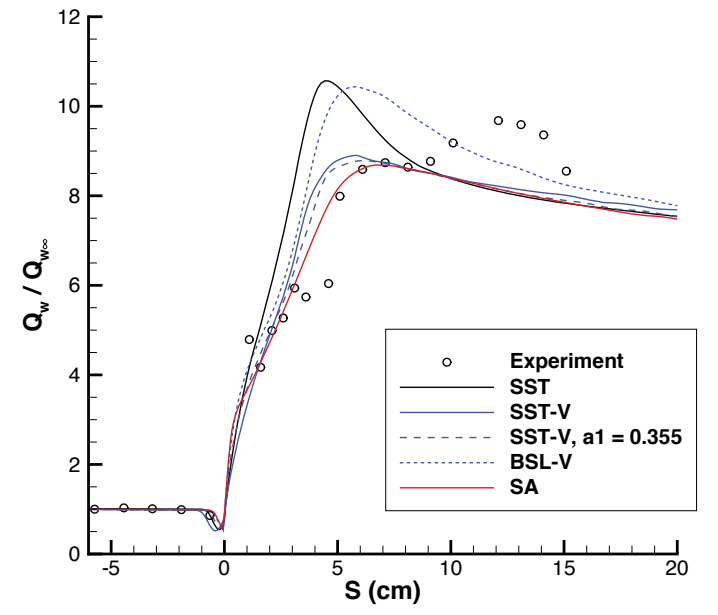

(b) Wall Heat Transfer

Figure 16. Comparison of surface quantities through SWBLI region, turbulence modeling effects.

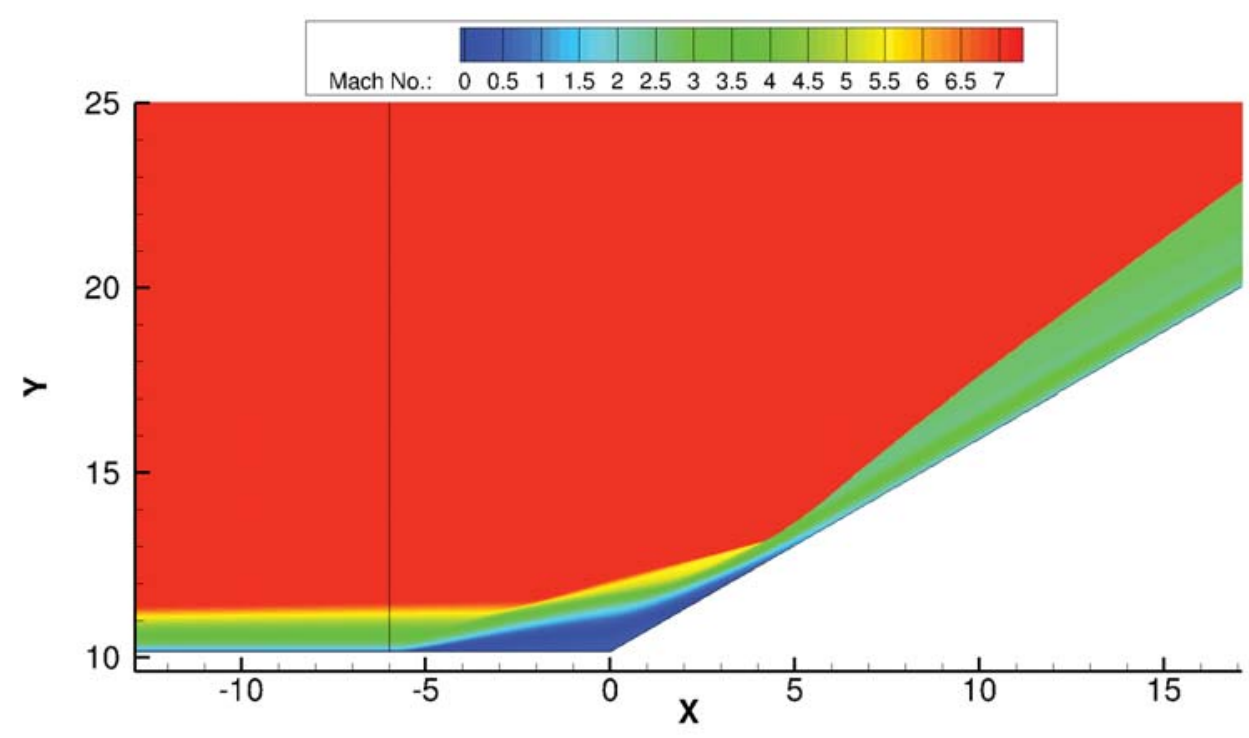

Figure 17. Closeup of Mach number contours (no-cone approach) near SWBLI focus region for 30-degree flare. 


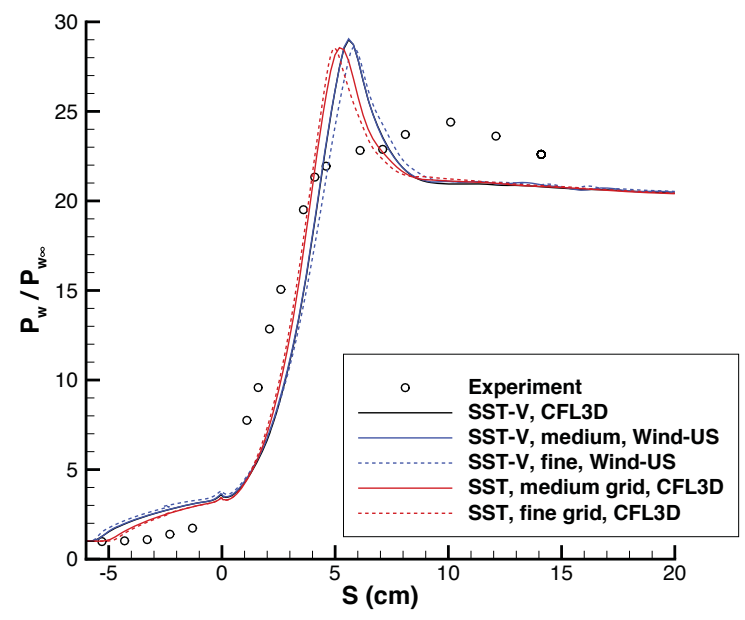

(a) Wall Pressures

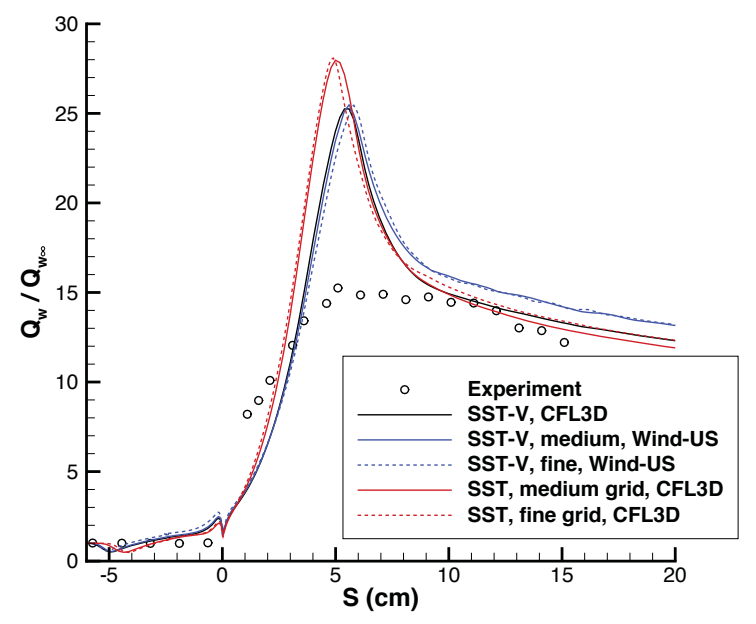

(b) Wall Heat Transfer

Figure 18. Comparison of surface quantities through SWBLI region, grid effects, 30-degree flare case.

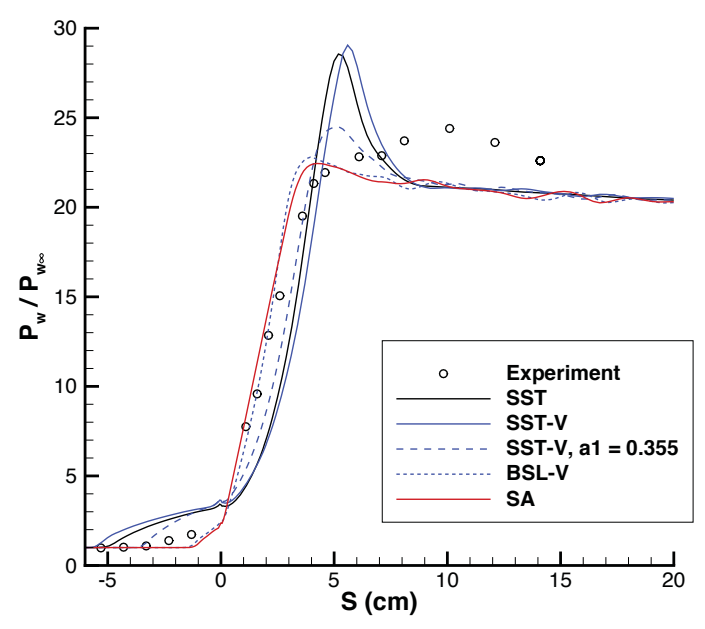

(a) Wall Pressures

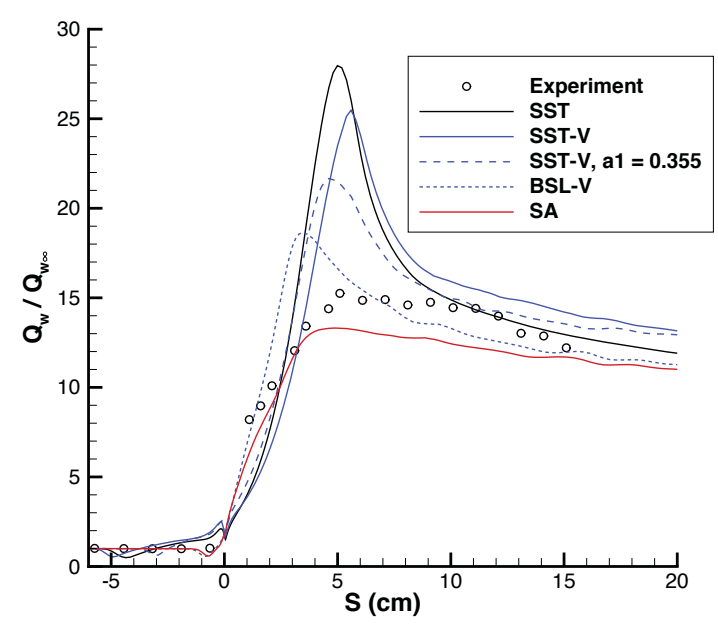

(b) Wall Heat Transfer

Figure 19. Comparison of surface quantities through SWBLI region, different turbulence models, 30-degree flare case. 